Submitted to Astrophysical Journal

Preprint typeset using IATEX style emulateapj v. 6/22/04

\title{
GAS FEEDBACK ON STELLAR BAR EVOLUTION
}

\author{
Ingo Berentzen $^{1}$, IsaAC Shlosman and Inma Martinez-Valpuesta ${ }^{2,3}$ \\ Department of Physics and Astronomy, University of Kentucky, Lexington, KY 40506-0055, USA \\ email: iberent@pa.uky.edu, shlosman@pa.uky.edu, martinez@pa.uky.edu \\ AND \\ Clayton H. Heller \\ Department of Physics, Georgia Southern University, Statesboro, GA 30460, USA \\ email: cheller@georgiasouthern.edu \\ Submitted to Astrophysical Journal
}

\begin{abstract}
We have analyzed evolution of live disk-halo systems in the presence of various fractions of gas, $f_{\text {gas }} \leq 8 \%$ of the disk mass, for 5 Gyr. Specifically, we have addressed the issue of angular momentum $(J)$ transfer from the gas to the stellar bar and its effect on the bar evolution. We find that the weakening of the bar over this time period, reported in the literature, is not related to the $J$-exchange with the gas, but is caused by the vertical buckling instability in the gas-poor disks and by a steep heating of a stellar velocity dispersion by the central mass concentration (CMC) in the gas-rich disks. On the other hand, the gas has a profound effect on the onset of the buckling - larger $f_{\text {gas }}$ brings it forth due to the more massive CMCs. The former process leads to the well-known formation of the boxy/peanut-shaped bulges, while the latter results in the formation of progressively more elliptical bulges, for larger $f_{\text {gas }}$. The subsequent (secular) evolution of the bar differs - the gas-poor models exhibit a growing bar while gas-rich models show a declining bar whose vertical swelling is driven by a secular resonance heating. The border line between the gas-poor and -rich models lies at $f_{\text {gas }} \sim 3 \%$ in our models, but is model-dependent and will be affected by additional processes, like star formation and feedback from stellar evolution. The overall effect of the gas on the dynamical and secular evolution of the bar is not in a direct $J$ transfer to the stars, but in the loss of $J$ by the gas and its influx to the center that increases the CMC. The more massive CMC damps the vertical buckling instability in the bar and depopulates orbits responsible for the appearance of boxy/peanut-shaped bulges. The combined action of resonant and non-resonant processes in gas-poor and gas-rich disks leads to a converging evolution in the vertical extent of the bar and its stellar dispersion velocities, and to a diverging evolution in the bulge properties.

Subject headings: galaxies: bulges - galaxies: evolution - galaxies: formation - galaxies: halos galaxies: kinematics and dynamics - galaxies: structure
\end{abstract}

\section{INTRODUCTION}

Galactic bars break the axial symmetry of rotating disks in the most profound way because they are sufficiently massive - thus being of a paramount importance to the short and long term galaxy evolution. However, various processes associated with bars are still poorly understood. Barred galaxies consist of stellar disks embedded in the dark matter (DM) halos with an admixture of gas. This gas is deprived of the rotational support by the bar and is channeled towards the central regions of few $\times 100 \mathrm{pc}-1 \mathrm{kpc}$, accumulating there in the form of the Central Mass Concentration (CMC), that includes gas, stars and a certain amount of the DM, and typically harbors the central supermassive black hole (SBH). Our main goal is to analyze the changes in the stellar bar as a result of this process - in other words to study the gas feedback on the bar evolution.

In self-gravitating systems with a dynamically significant rotation, any substantial departure from axial symmetry is destined to speed up the evolution, leading to

\footnotetext{
${ }^{1}$ Present address: Astronomisches Rechen-Institut, Zentrum für Astronomie, D-69120 Heidelberg, Germany

${ }^{2}$ Gruber Foundation Fellow at OAMP, 13004, Marseille, France

${ }^{3}$ Present address: Instituto de Astrofisica de Canarias, E-38200, La Laguna, Tenerife, Spain
}

mass and angular momentum redistribution in the system. While the role of the angular momentum in this process has been emphasized already by Lynden-Bell \& Kalnajs (1972), the efficiency of this transport and dependence on various parameters is still under investigation (e.g., Athanassoula 2003). Tremaine \& Weinberg (1984) and Weinberg (1985) have estimated that the bar should lose most of its momentum to the surrounding DM halo in a few rotations $(\sim 1 \mathrm{Gyr})$ - this was not supported by subsequent numerical simulations (Sellwood 2006), but the overall trend, that the angular momentum flows from the inner, bar-unstable disk to the outer disk and to the halo, has been confirmed.

Our view on the role of a DM halo in disk galaxy evolution has changed dramatically over the last few years - from the original claim that it damps the bar instability (e.g., Ostriker \& Peebles 1973), to recent results that more massive halos grow larger bars (e.g., Athanassoula \& Misiriotis 2002). It is a common wisdom today that bars on all scales redistribute mass and angular momentum in the main body of the galaxy and alter the radial profiles of gas, stars and DM densities. In the long run, the DM halos serve as a sink for the angular momentum from the disk and this process is mediated by the bars.

Disk galaxies possess various amounts of a cold gas, 
typically $<10 \%$ of the disk mass, and probably have been more gas-rich in the past. However, the ability of the gas to influence the galactic dynamics of the parent object extends well beyond its mass fraction. The gas dissipates and therefore can form bound massive accumulations even in excess of $10^{7} \mathrm{M}_{\odot}$. In this sense the gas can be more clumpy than a collisionless matter, either stellar or DM. This leads to a number of dynamical consequences, more importantly to a dynamical friction and to scattering and randomizing of stellar and DM particle orbits (Shlosman \& Noguchi 1993). The angular momentum redistribution in numerical simulations with gas has been examined by Berentzen al. (1998) and Berentzen et al. (2004).

Furthermore, the gas bears similar amounts of a specific angular momentum with stars in the cold disk prior to the bar instability. Because of its viscosity, the gas responds to a bar-like perturbation with a phase shift, compared to the stellar response. This leads to streamline intersections and to shocks downstreams from the bar major axis. Resulting leading dust lanes delineate the underlying shocks and associated density enhancements (e.g., Athanassoula 1992). The gravitational torques from the bar extract the angular momentum from the gas and transfer it to the underlying stellar (and DM) component which lags behind the gas. These torques depend on the shift in the position angle between the gas and stellar distributions in the bar,

$$
\text { Torque }=-\left[\int_{0}^{2 \pi} \int_{0}^{\infty} \Sigma^{\mathrm{a}}(r, \phi) \frac{\partial \Phi_{\mathrm{gas}}^{\mathrm{a}}(r, \phi)}{\partial \phi}\right] r d r d \phi,
$$

where only the asymmetric part of the gas gravitational potential $\Phi_{\text {gas }}^{\mathrm{a}}$ which acts on the segment $d r$ of the asymmetric stellar density distribution $\Sigma^{\mathrm{a}}(r, \phi)$ in the cylindrical system of coordinates $r, \phi$, will make a non-zero contribution.

As a result, the gas falls towards the central kpc where the bar potential is again more axisymmetric and the resulting shock focusing injects the gas onto the weakly elliptical orbits in situ, forming nuclear rings. These rings are ubiquitous in barred galaxies (Buta \& Combes 1996; Knapen 2005), but their subsequent evolution can differ (Knapen et al. 1995; Heller \& Shlosman 1996; Heller, Shlosman \& Englmaier 2001; Englmaier \& Shlosman 2004). The ultimate fate of the inflowing gas is debatable, but there is a strong theoretical and observational evidence that some of this gas can reach deep inside the central region and fuel the nuclear star formation and the accretion processes onto the central SBH as a result of gravitational instabilities in the gas itself (e.g., Shlosman, Frank \& Begelman 1989; Shlosman, Begelman \& Frank 1990; Ishizuki et al. 1990; Kenney et al. 1992; Forbes et al. 1994; Knapen et al. 1995; Maiolino et al. 2000; Jogee et al. 2002; Shlosman 2005; Jogee 2006), and contribute to the formation of the $\mathrm{BH}$ itself (Begelman, Volonteri \& Rees 2006; Heller, Shlosman \& Athanassoula 2007).

Whether the inflowing gas fuels the central SBH, or contributes to the buildup of stellar bulges (Kormendy \& Kennicutt 2004 and refs. therein), the growing CMC in barred galaxies has been reported to have a strong effect on the stellar bar. Hasan \& Norman (1990), Friedli (1994), Norman, Sellwood \& Hasan (1996), Berentzen et al. (1998), Shen \& Sellwood (2004), and others have observed that the bar dissolves only when massive and compact CMCs form - a process that is affected by the bar structure and its host DM halo (Athanassoula, Lambert \& Dehnen 2005). Such extreme CMCs can be represented only by the SBHs. However, the required $\mathrm{SBH}$ mass of a few percent of the disk mass is more than a factor of ten larger than the SBH masses found in disk galaxies (e.g., Ferrarese \& Ford 2005). Hence the stellar bars are much more resilient than previously envisioned.

Furthermore, Bournaud, Combes \& Semelin (2005) have argued, based on numerical simulations of $7.25 \%$ gas-rich disks embedded in rigid halos, that the gas is able to weaken the stellar bars dramatically, even before the $\mathrm{CMC}$ is in place - the reason for this is the transfer of angular momentum from the gas to the bar. The combination of angular momentum transfer to the stars and the subsequent buildup of the CMC destroy the bar over the timescale as short as 1.4 Gyr — claimed by Bournaud et al.

Here we analyze the bar evolution for various gas fractions in the disk and allow for the growth of the CMC and SBH. Unlike Bournaud et al. (2005), we use a disk immersed in the live DM halo. In addition to the standard model, we supplement our analysis with a number of test models. We limit the discussion to 5 Gyr of bar evolution in order to make a direct comparison with Bournaud et al. This paper is structured as following. Section 2 describes our numerical tools and the initial conditions used in this work. Section 3 provides the results and Section 4 presents a number of test models. We conclude with the discussion in Section 5 .

\section{NUMERICAL METHOD AND INITIAL CONDITIONS}

We use the updated hybrid $N$-body and Smooth Particle hydrodynamics (SPH) code of Heller \& Shlosman (1994). The version FTM-4.4 of the code uses the FalcON force solver of Dehnen (2002) — a tree code with mutual cell-cell interactions and complexity $O(N)$. It conserves the momentum exactly and is about ten times faster than the optimally-coded Barnes \& Hut (1986) tree code. We use a constant gravitational softening of $160 \mathrm{pc}$ for the gas and for the collisionless particles. The amount of DM particles is $N_{\mathrm{DM}}=1.2 \times 10^{5}$, stellar particles $N_{*}=3.6 \times 10^{5}$, and gas $N_{\text {gas }}=4 \times 10^{4}$. The DM particle mass is $1.1 \times 10^{6} \mathrm{M}_{\odot}$, the stellar particle is $1.5 \times 10^{5} \mathrm{M}_{\odot}$, and the gas particle is $1.2 \times 10^{5} \mathrm{M}_{\odot}$ for the gas fraction $f_{\text {gas }}=8 \%$ of the disk mass. Models with a much larger number of the disk and DM particles, making DM/stellar mass ratio per particle $\sim 1$, did not change the evolution over the run times of our models. The energy and angular momentum concervation in the pure collisionless models is better than $0.15 \%$ and $0.02 \%$, correspondingly.

For models with SBHs, the central SBH evolution is given by a single stellar particle with initially a small 'seed' mass of $10^{5} \mathrm{M}_{\odot}$. It is nailed to the position of the center-of-mass (CoM) of the system at $\tau=0$ and has a fixed gravitational softening $\epsilon_{\bullet}=160 \mathrm{pc}$ (a Plummer sphere with a characteristic $r_{0}=160 \mathrm{pc}$ ), except in one model (G0BH80, see Table 2) where $\epsilon_{\bullet}=80 \mathrm{pc}$. The CoMs of the disk and the halo stay within $\sim 50 \mathrm{pc}$ from the $\mathrm{SBH}$, damping each others motions. We find no differences in the behavior of the CoMs in the models 
TABLE 1

G0: Standard Model Parameters

\begin{tabular}{|c|c|c|c|c|}
\hline Parameters & Stellar Disk & DM Halo & $\mathrm{SBH}$ & Notes \\
\hline Radial scalelength & $2.85 \mathrm{kpc}$ & - & - & \\
\hline Vertical scalelength & $0.2 \mathrm{kpc}$ & - & - & \\
\hline Total mass & $0.58 \times 10^{11} \mathrm{M}_{\odot}$ & $1.33 \times 10^{11} \mathrm{M}_{\odot}$ & 0 & \\
\hline Mass $(<10 \mathrm{kpc})$ & $0.5 \times 10^{11} \mathrm{M}_{\odot}$ & $0.5 \times 10^{11} \mathrm{M}_{\odot}$ & 0 & \\
\hline Gas fraction $\left(f_{\text {gas }}\right)$ & 0 & 0 & - & \\
\hline
\end{tabular}

TABLE 2

List of Models

\begin{tabular}{|c|c|c|c|c|c|}
\hline Model & $f_{\text {gas }}(\%)$ & SBH properties & Q & Main Figs. & Notes \\
\hline G0 & 0 & - & 1.5 & $1 \mathrm{a}, \mathrm{b}$ & Standard Model: DM + stellar disk \\
\hline G05 & 0.5 & - & 1.5 & $1 \mathrm{a}, \mathrm{b} ; 2$ & Standard Model with $0.5 \%$ gas \\
\hline G2 & 2 & - & 1.5 & & as G05 with $2 \%$ gas \\
\hline G4 & 4 & - & 1.5 & & as G05 with $4 \%$ gas \\
\hline G6 & 6 & - & 1.5 & & as G05 with $6 \%$ gas \\
\hline G8 & 8 & - & 1.5 & & as G05 with $8 \%$ gas \\
\hline G05BH & 0.5 & growing & 1.5 & $1 \mathrm{a}, \mathrm{b} ; 3$ & as G05 but with a growing SBH \\
\hline G2BH & 2 & growing & 1.5 & & as $\mathrm{G} 2$ but with a growing $\mathrm{SBH}$ \\
\hline G4BH & 4 & growing & 1.5 & & as G4 but with a growing $\mathrm{SBH}$ \\
\hline G6BH & 6 & growing & 1.5 & & as G6 but with a growing $\mathrm{SBH}$ \\
\hline G8BH & 8 & growing & 1.5 & & as G8 but with a growing $\mathrm{SBH}$ \\
\hline G8-25T & 8 & growing & 1.5 & 4 & $25 \%$ gas torques removed \\
\hline G8-50T & 8 & growing & 1.5 & & $50 \%$ gas torques removed \\
\hline G8-75T & 8 & growing & 1.5 & & $75 \%$ gas torques removed \\
\hline G8-100T & 8 & growing & 1.5 & & $100 \%$ gas torques removed \\
\hline G0GT & & - & 1.5 & 5 & $\begin{array}{l}\text { no gas, adding 'quasi-exact' gas force field in } \\
\text { form of an external potential from G8BH }\end{array}$ \\
\hline G0BH & 0 & growing & 1.5 & 6 & using G8BH model for the SBH growth \\
\hline G0BH250 & 0 & growing & 1.5 & 6 & $\begin{array}{l}\text { using G8BH model for the } \mathrm{SBH} \text { growth and gas } \\
\text { inside } r_{0}=250 \mathrm{pc} \text { added to the } \mathrm{SBH}\end{array}$ \\
\hline G0BH80 & 0 & growing & 1.5 & 7 & $\begin{array}{l}\text { using G8BH model: SBH mass incl. gas within } 250 \text { pc. } \\
\text { The only model that has } \epsilon_{\bullet}=80 \mathrm{pc}\end{array}$ \\
\hline G0BH750 & 0 & growing & 1.5 & 7 & $\begin{array}{l}\text { using } \mathrm{G} 8 \mathrm{BH} \text { for the SBH and gas (Plummer sphere } \\
\text { within } r_{0}=750 \mathrm{pc} \text { ) growth }\end{array}$ \\
\hline G0-Q18 & 0 & - & 1.8 & 8 & as G0 but with $Q=1.8$ \\
\hline G8BH-Q18 & 8 & growing & 1.8 & 8 & as $\mathrm{G} 8 \mathrm{BH}$ but with $Q=1.8$ \\
\hline
\end{tabular}

Note. - Columns: (1) model type (see text); (2) gas fraction (\%) of the disk mass; (3) "growing" — growing the BH from a seed value of $10^{5} \mathrm{M}_{\odot}$; (4) initial value of $Q$ parameter; (5) main figure(s) introducing this model; (6) comments

with and without the gas. The accretion radius of the $\mathrm{SBH}$ is taken as $R_{\mathrm{acc}}=40 \mathrm{pc}$. Particles which are found within this radius and which are bound to the $\mathrm{SBH}$, are extracted from the simulations and their mass is added to the $\mathrm{SBH}$. The requirement for a small timestep near the CMC and the central SBH are satisfied by the hierarchical timesteps in the FTM code. We typically use between nine to twelve time bins, each differing by a factor of 2 .

The initial conditions (see Table 1) have been obtained iteratively from Fall \& Efstathiou (1980) to assemble disk and halo particles in a virial equilibrium (see Heller \& Shlosman 1994 for more details). The halo has been relaxed in the frozen disk potential for $\Delta \tau \sim 2.4 \mathrm{Gyr}$. The disk is axisymmetric with the Toomre's parameter $Q=1.5$ for the stars only, and the halo-to-disk mass ratio is $\sim 1$ within $R=10 \mathrm{kpc}$, at the start of the simulations. Other values of $Q$ are used as well, and the asymmetric drift is accounted for. When the gas is added to the disk, the total disk mass is kept unchanged. We use the isothermal equation of state (EOS) with the temperature of $T=10^{4} \mathrm{~K}$ but have run also models with the adiabatic EOS - no differences have been detected. The gas has the stellar radial distribution initially, vertically it is in a hydrostatic equilibrium. The pure collisionless model parameters are listed in Table 1 and the list of all the models with gas and additional test models are listed in Table 2. Overall, our initial conditions are identical to those used by Berentzen et al. (1998), but the numerical resolution in the current models is much superior. This allows us to compare the models and be guided by Berentzen et al. nonlinear orbit analysis and their usage of the Poincare sections in dissecting the models.

\section{RESULTS: VARYING THE GAS FRACTION}

Our benchmark model G0 is a pure collisionless (DM+stars) model which shows a rapidly growing stellar bar whose $m=2$ normalized amplitude $A_{2}$ of the stellar component saturates at $\tau \sim 1.2$ Gyr. We define $A_{2}$ within a cylindrical region of $0 \leq r \leq 7.5 \mathrm{kpc}$ and 

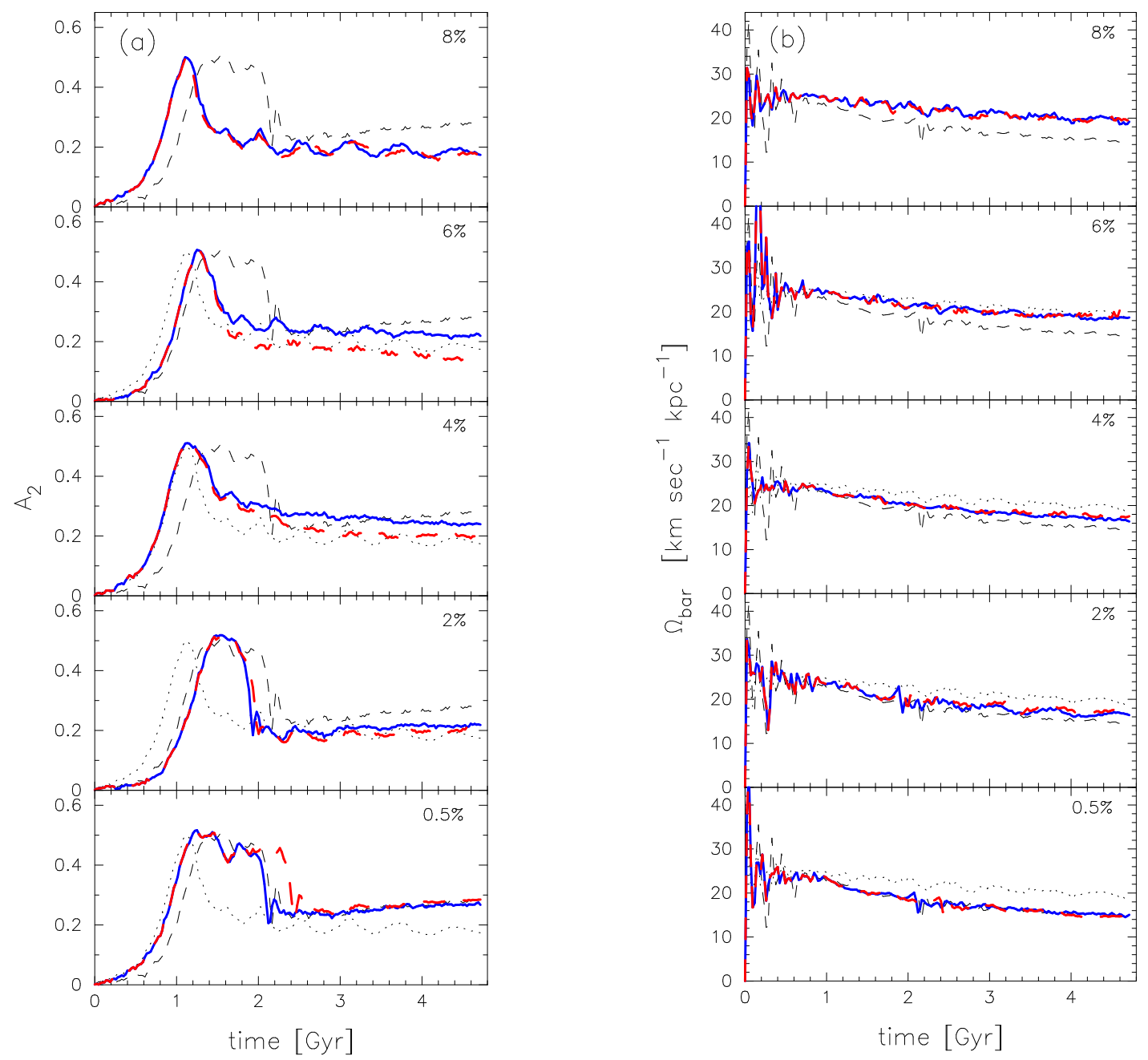

FIG. 1. - Evolution of (a) stellar bar amplitude $A_{2}$ and $(b)$ the bar pattern speed $\Omega_{\mathrm{bar}}$ (in $\mathrm{km} \mathrm{sec}^{-1} \mathrm{kpc}^{-1}$ ) given by the $m=2$ mode in models (from the bottom to the top) G05-G8 (blue lines), with various $f_{\text {gas }}$, superposed on the G05BH-G8BH (red dashed lines) with a growing SBH, and on the collisionless model G0 (black dashed line) over the first 5 Gyr. For a comparison, we also display G8 model (dotted black lines).

$|z| \leq 1 \mathrm{kpc}$, so it encompasses the modeled stellar bars at nearly all times, and normalize it by the amplitude of the $m=0$ term. After the extended plateau of about 0.9 Gyr, this amplitude drops sharply to about $A_{2} \sim 0.2$. The following evolution of the bar is that of a gradual strengthening (Fig. 1a).

Models with $f_{\text {gas }}=0.5 \%-8 \%$ gas, G05-G8 (Fig. 1a), without the central $\mathrm{BH}$, show a similar rise and drop as G0 in the bar strength. The main differences appear to be the existence of an extended plateau before the drop in $A_{2}$, which gradually disappears in models with larger gas fraction, for $\gtrsim 4 \%$, and the maximum in $A_{2}$ which is reached slightly earlier for these models. The behavior of $A_{2}$ near its maximum changes from G05 to G8 gradually - in the gas poor models the bar evolution converges to that in G0. Following the large drop, $A_{2}$ changes little over the time of the simulations: G0-G2 show a slight increase while G4-G8 models show a slight decrease. Here we focus on the sharp drops in $A_{2}$ that are visible in all models.

The bar pattern speeds, $\Omega_{\mathrm{bar}}$, are shown in Fig. $1 \mathrm{~b}$. Models with larger $f_{\text {gas }}$ slow down more gradually than the gas-poor models, by about $30 \%$. There is also a substantial difference between the behavior of the corotation $(\mathrm{CR})$ radius in gas-rich and gas-poor models. In G0, the $\mathrm{CR}$ increases from $\sim 9 \mathrm{kpc}$ to $14 \mathrm{kpc}$, while that of $\mathrm{G} 8$ model stays flat initially and then increases negligibly to $10 \mathrm{kpc}$ over the simulation time of 5 Gyr.

The CMCs which correspond to a total mass accumulation within the central $250 \mathrm{pc}$, as well as their gaseous and stellar components, for models G05-G8, have been displayed in Fig. 2. The pure stellar model G0 does not grow a visible CMC, while other models grow it during $0.2-1.5 \mathrm{Gyr}$, with longest timescale corresponding to the most gas-poor models. The growth period can be roughly divided into the initial shallow growth - this is more prolonged for models with lower $f_{\text {gas }}$, and the second, avalanche-type growth, that is $\sim 0.15$ Gyr for all models. The peak growth rate of the $\mathrm{CMC}$ is attained around the peak of the bar strength.

\subsection{Results: Adding the Central BH}

In models $\mathrm{G} 05 \mathrm{BH}-\mathrm{G} 8 \mathrm{BH}$, the bar-triggered radial gas inflow leads to the gas accumulation in the central region 


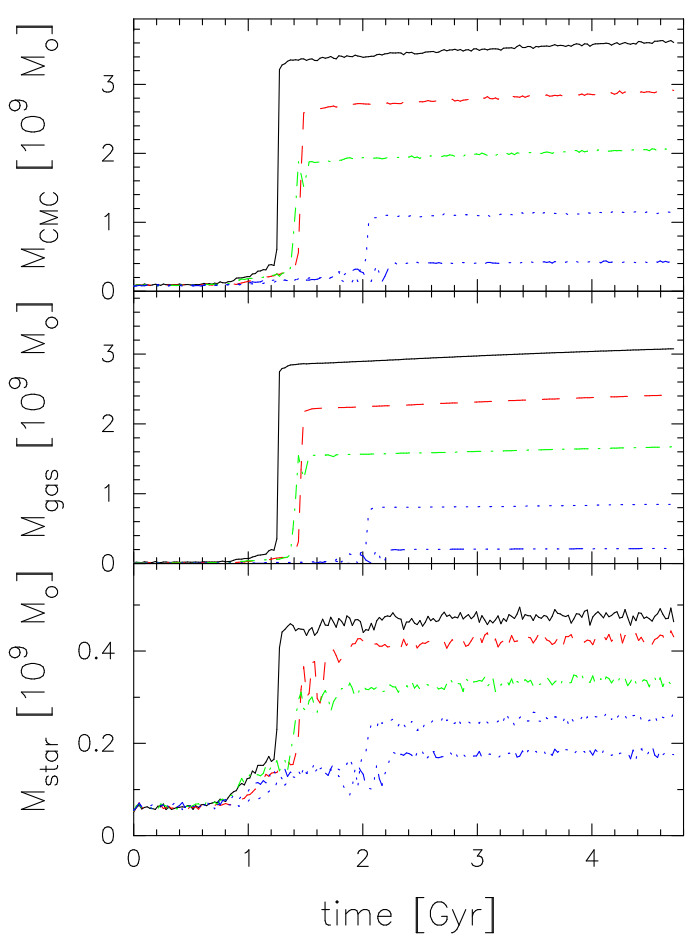

Fig. 2. - Growth of the CMC in G05 - G8 models within the central 250 pc. Top: total (CMC) mass within this radius; Middle: gas mass; Bottom: stellar mass. The colors are: G05 (blue dashed-dot-dot-dotted), G2 (blue dotted), G4 (green-dotted), G6 (red-dashed) and G8 (solid black).

as well as in the fueling of the central BH surrounded by gas. Figs. 2 and 3 demonstrate both the growth of the $\mathrm{BH}$ mass, $M_{\bullet}(\tau)$, and that of the $\mathrm{CMC}$ within the central $250 \mathrm{pc}, M_{\mathrm{CMC} 250}(\tau)$, which includes the $\mathrm{BH}$, gas, stars and DM, as a function of time. Subsequently to the bar decay from its maximal strength, the growth of $M_{\bullet}(\tau)$ and of $M_{\mathrm{CMC} 250}(\tau)$ saturate within $\lesssim 0.2$ Gyr, i.e., almost instantly. The subsequent evolution of the CMC and the $\mathrm{BH}$ is very mild. The final $M_{\bullet}$ and $M_{\mathrm{CMC} 250}$ scale linearly with the initial gas fraction in the disk. A fraction $\sim 65 \%-75 \%$ of the gas ends up in the CMC in all models with the $\mathrm{BH}$ - for smaller $f_{\text {gas }}$ there is fractionally more gas in the end (Fig. 3). Overall, we do not find a substantial difference between the CMCs in models with and without the central BH. However, models with larger $f_{\text {gas }}$ grow more massive $\mathrm{BHs}$ and CMCs affecting the subsequent bar evolution, both its vertical and planar structures, as we discuss in Section 5.

\section{TESTING THE MODELS}

Models with an increasing gas fraction, both with and without the $\mathrm{BH}$, exhibit continuity of bar properties. All of them develop bars of nearly identical strength when measured by the $A_{2}$ amplitude. Even more spectacular is the subsequent decrease in the bar strength as shown in Fig. 1. While the shape of $A_{2}(\tau)$ differs among the models, the amount of the post-maximum drop is the same. Because the models differ in the gas fraction, $f_{\text {gas }}$, the evolution of the CMC and of the central $\mathrm{BH}$ will differ as well. Comparison of Figs. 1-3 shows that the presence of the SBH has only a minimal influence on the bar strength, its pattern speed, and on the CMC. We, there-

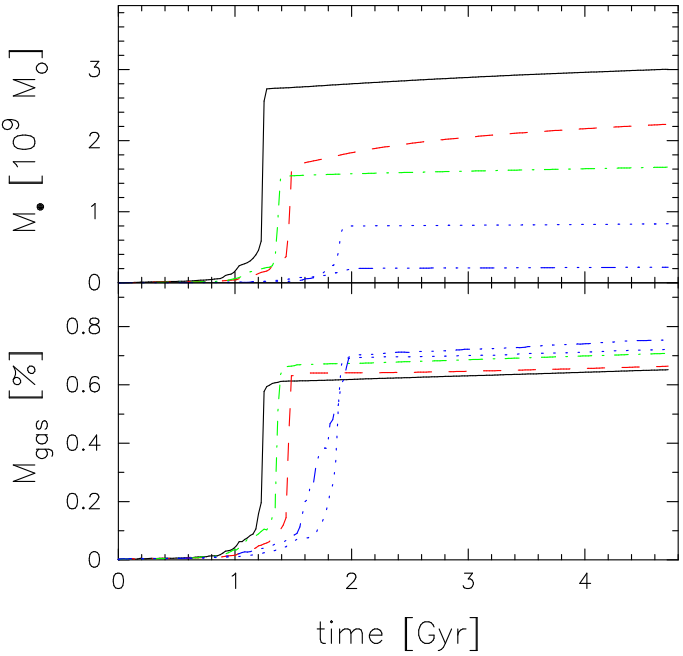

FIG. 3.- Growth of the central BH (upper frame) in G05BH G8BH models, and evolution of the gas fraction of the total CMC mass within the central $250 \mathrm{pc}$ (lower frame). The mass of the $\mathrm{BH}$ is counted as contributing to the gas mass. The colors are: G05BH (blue dotted), G2BH (blue dashed-dot-dot-dotted), G4BH (green dash-dotted), G6BH (red-dashed) and G8BH (solid black).

fore, consider the models with the $\mathrm{BH}$ as representative and base our discussion on them. Difference between the models is mentioned only when it is substantial.

\subsection{Removing gas gravitational torques on stars}

The stellar and gas fluids differ in their intrinsic physical properties, specifically in viscosity, which generates the time lag in the gas response to any perturbation. This time lag is the source of gas-stars mutual gravitational torques within the bar (Eq. 1) - with no viscosity the contribution of this integral is zero. The angular momentum within the bar flows from the gas to the stars in the disk and to a certain degree to the DM halo. Removing these torques and comparing the models will allow for a direct testing of Bournaud et al. (2005) claim that they affect the stellar bar evolution.

Because we aim at understanding the effect of the gas on the bar evolution, specifically through angular momentum redistribution in the system, we use the bar strength measured by $A_{2}$ and follow the balance of the angular momentum in the basic morphological components - the disk, bar and the DM halo. We first ask the question, to what degree the gas is responsible for the bar weakening as shown in the Fig. 1a frames. We test the possibility that the large drop in the bar strength shown in various models of Fig. 1a between $\tau \sim 1-2.5 \mathrm{Gyr}$ results from the input of the angular momentum coming from the gas gravitational torques as proposed by Bournaud et al. (2005).

The fact that G0, the pure stellar model, shows the same qualitative behavior as other models and the degree of weakening is even quantitatively similar, rises serious doubts that the gas is in any way responsible for the bar downsizing. Nevertheless, we perform the first test of removing a fixed fraction of the gravitational torques, by removing the tangential components of the gravitational forces, applied by the gas on the stars and the DM. Fig. 4 exhibits four models based on G8BH where 


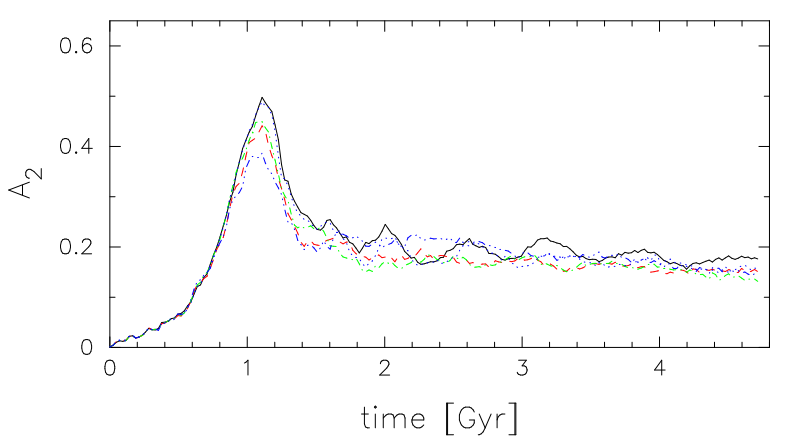

FIG. 4.- Evolution of G8 model with gradually subtracted torques from the gas onto the stars. Shown are models with $25 \%$ torques subtracted (black dotted), 50\% subtracted (green dashdotted), $75 \%$ subtracted (red dashed), $100 \%$ subtracted (blue dashdot-dot-dotted). For the comparison we also display G8 (black solid).

the torques have been reduced by $25 \%, 50 \%, 75 \%$ and $100 \%$, i.e., models G8-25T-100T. It shows that there is no substantial difference between the G8BH and G8T models. While the $A_{2}$ peak around 1.1 Gyr lowers slightly, when the torques are removed, we consider this to be a numerical rather than physical effect. For example, differences of this level are even expected for the same model that uses a different random 'seed' in the initial conditions. The subsequent evolution of models in Fig. 4 shows a clear convergence trend. While indeed the stellar bar receives less angular momentum from the gas, one is forced to conclude that the gas torques have no effect on the observed drop in the bar strength in our models. The question of course is what is the origin of this drop. We address this issue in Section 5 after exploring various venues via test models.

\subsection{Gas Substitutes}

In tandem with tests shown in Fig. 4, we advanced the G0 model with artificially added gravitational torques from the gas in G8BH model. For this purpose, we calculate the torques from the gaseous component on a cartesian grid for the (total) dimension of $50 \mathrm{kpc} \times 50 \mathrm{kpc}$ $\times 4 \mathrm{kpc}$. The (constant) grid spacing is $250 \mathrm{pc}$ along the $x$ - and $y$-axes and $2 \mathrm{kpc}$ along $z$. In order to have a smooth force field, we take time average over several frames. We bring up the force field quasi-adiabatically between $\tau \sim 0.94-1.22$ Gyr (Fig. 5), or over 20 dynamical times, reaching its full strength about the time when the bar amplitude also reaches its maximum. To apply the torques on the stellar and DM components, we use a $2 \mathrm{D}$ spline interpolation.

The resulting evolution (model G0GT) follows closely that of the G8BH model, with the exception of the $A_{2}$ drop time, i.e., the extent of the associated plateau. Both tests performed in this section agree with our previous conclusion that, for models with $f_{\text {gas }} \lesssim 8 \%$, the gravitational torques from the gas do not alter substantially the drop in the $A_{2}$ observed in all models, but have a profound effect on the extent of the plateau which precedes the drop. They also serve as an independent verification that our modeling of effects of the gas component are sufficiently reasonable and do not alter the model evolution in some unexpected way.

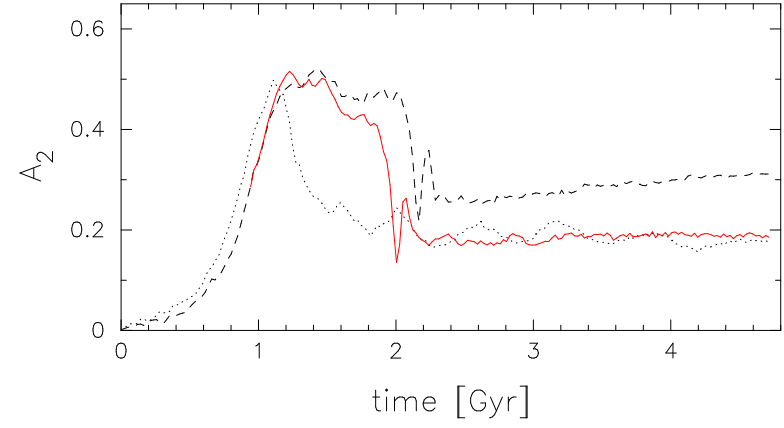

FIG. 5.- Evolution of $A_{2}$ in the G0GT model (solid red): constructed from the G0 model under the gravitational torques from the gas onto the stars in G8BH. For the comparison we also display G8BH (black dotted) and G0 (black dashed).

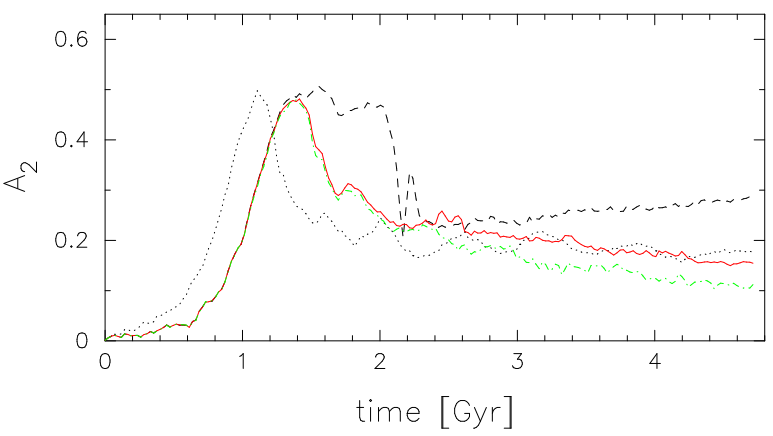

FIG. 6.- Evolution of $A_{2}$ in (a) G0BH model with an artificially grown $\mathrm{BH}$, whose history is taken from G8BH (red line), and (b) G0BH250 model with an artificially grown $\mathrm{BH}$ and the gas within the central $250 \mathrm{pc}$ (added to the BH mass) (green dash-dotted). G0 and G8BH curves were added for a comparison.

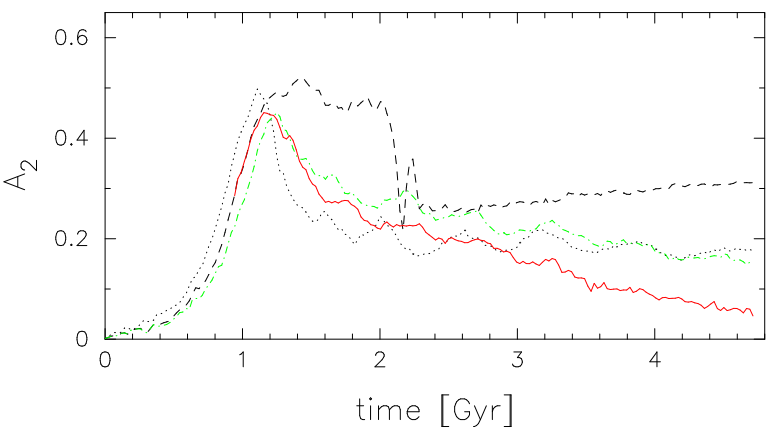

Fig. 7.- Evolution of $A_{2}$ in (a) G0BH80 model with an artificially grown $\mathrm{BH}$ with a smaller gravitational softening of $80 \mathrm{pc}$, whose history is taken from G8BH and is a combined mass of the $\mathrm{SBH}$ and the gas within the central $250 \mathrm{pc}$ (red line), and (b) G0BH750 model with an artificially grown BH (regular softening of $160 \mathrm{pc}$ ) and the gas within the central $r_{0}=750 \mathrm{pc}$ which is modeled as a Plummer sphere with $r_{0}$ (green dash-dotted). G0 and $\mathrm{G} 8 \mathrm{BH}$ curves were added for a comparison.

\subsection{Artificial growth of the $B H$ and the $C M C$}

The stellar bar is expected to evolve with respect to the buildup of the central $\mathrm{BH}$ and the nearby gas accumulation. If these grow on a secular timescale, i.e. adiabatically, this gas will drag in additional stars and DM. The evolution of the mass within the central 250 pc and the central SBH are shown in Figs. 2 and 3 for 
models with various $f_{\text {gas }}$.

To further isolate the consequences of a gas influx towards the central regions, we have used the pure stellar model G0 and imposed the BH and gas accumulation histories taken from a gas-rich model. The CMC is defined as earlier and consists of the gas accumulation within the central $250 \mathrm{pc}$ (or $750 \mathrm{pc}$ ), stars and DM there. Fig. 6 shows two such models: $(a)$ an artificially growing $\mathrm{BH}$ taken from the $\mathrm{G} 8 \mathrm{BH}$ (model $\mathrm{G0BH}$ ); $(b)$ an additional model of a growing $\mathrm{BH}$ from $\mathrm{G} 8 \mathrm{BH}$ when the gas within the central $250 \mathrm{pc}$ is added to the $\mathrm{BH}$ mass model (G0BH250). In both cases the gravitational softening of the $\mathrm{BH}$ is $160 \mathrm{pc}$ - the typical softening in our models. The model G0BH closely follows the corresponding G8BH, although no gravitational torques from the gas are present here. The $A_{2}$ curve has switched gradually from that of G0 to G8BH. The corresponding model G0BH250 with more massive SBH (as the gas within the central $250 \mathrm{pc}$ has been added to the $\mathrm{BH}$ ) falls below the G0BH curve, as expected - this confirms that more massive SBHs, albeit not found in disk galaxies by a large margin, can in fact dissolve the stellar bars, as discussed in the literature.

Fig. 7 displays $(a)$ the evolution a $\mathrm{BH}$ with an added gas mass from the central $250 \mathrm{pc}$ but the gravitational softening of the $\mathrm{BH}$ is decreased to $80 \mathrm{pc}$ (model G0BH80), to test a more compact mass distribution; and (b) a growing $\mathrm{BH}$ and a independently growing the gas accumulation within the central 750 pc (model G0BH750). This gas is approximated by a second Plummer sphere with a characteristic radius of $750 \mathrm{pc}$. The G0BH80 bar decays gradually and its $A_{2}$ fall below that of G0BH250 - smaller gravitational softening for the $\mathrm{BH}$ creates a more compact $\mathrm{CMC}$ here which starts to affect the bar secularly. The substantially more massive CMC in G0BH750 has a profound effect on the bar by dissolving it during $\sim 5 \mathrm{Gyr}$, as discussed in section 1 .

\subsection{Stellar Bars in Hot Disks}

Stellar bar instability is delayed and its amplitude is lowered in hotter disks, i.e., disks with larger stellar dispersion velocities (e.g., Athanassoula \& Sellwood 1983). In order to test this effect on the abrupt weakening of the bar in our models, we have increased the initial $Q$ parameter to 1.8 in G0 and G8BH models, hereafter models G0-Q18 and G8BH-Q18. Fig. 8 displays the evolution of these models. The risetime of the bar instability has increased as expected in both models, and the $A_{2}$ peaks are delayed with respect to the original models. The maxima of the $A_{2}$ amplitudes have been also lowered by $\sim 0.1$, and the amplitude of the drop in $A_{2}$ has diminished by this amount as well. The difference between the above models after the amplitude drop decreases substantially. The stellar bars survive and do not show any sign of decay over the simulation time of 5 Gyr.

\subsection{Angular Momentum Evolution}

Next, we analyze the angular momentum $(J)$ redistribution between the disk and the DM halo in the presence of the gas. The resonant interaction between the various morphological components will be addressed elsewhere. We have divided the disk/halo system into the (cylindrical) part within the CR and outside it. The overall $J$ of the DM halo increases sharply only after the bar has

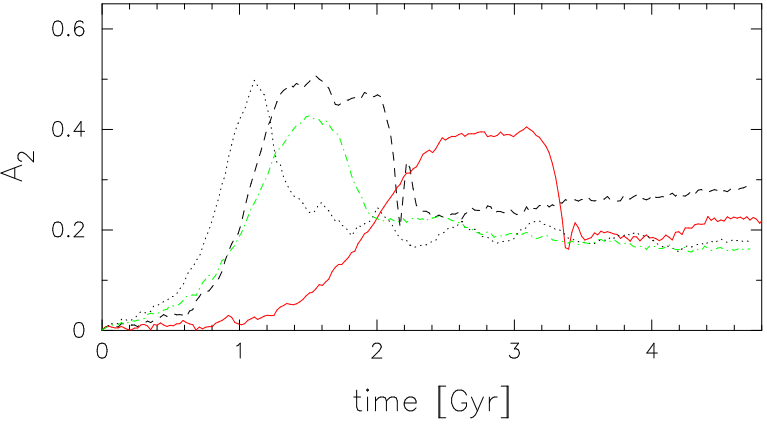

FIG. 8.- Evolution of $A_{2}$ in G0-Q18 (red line) and G8BH-Q18 (green dash-dotted) models which are similar to $\mathrm{G} 0$ and $\mathrm{G} 8 \mathrm{BH}$ but with $Q=1.8$. For the comparison we also display $\mathrm{G} 8 \mathrm{BH}$ (black dotted) and G0 (black dashed).

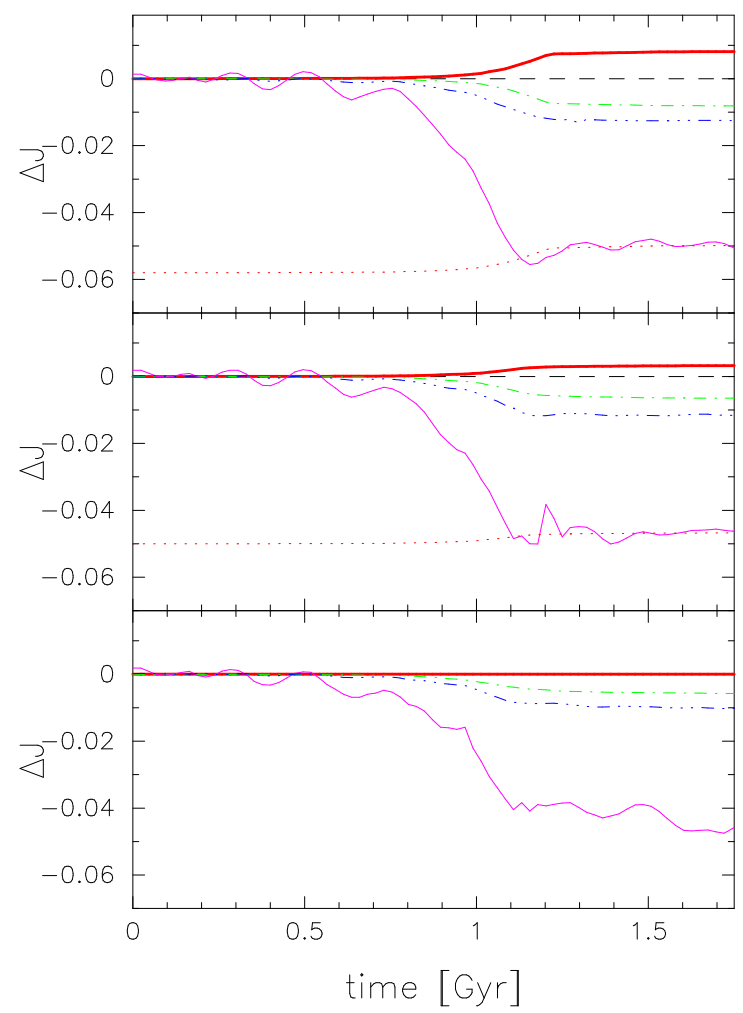

FIG. 9.- Change in the angular momentum $(\Delta J)$ within the CR radius in G8BH (top), G8-50T (center) and G8-100T (bottom). The solid red lines show $J$ flow from the gas to the stars calculated from integration of corresponding gravitational torques. The dashdotted green lines provide the $J$ flow from the stars to the gas, calculated from the torques as well. The dash-dot-dot-dotted blue and solid pink lines exhibit the $J$ flow in the gaseous and stellar component, respectively, calculated directly from model evolution. The red dotted lines are identical to the red solid lines shifted to match the pink lines. The black dashed line is added to emphasize the $\Delta J=0$ line.

reached it maximal amplitude, i.e., after $\sim 1$ Gyr and is larger by a factor of $\sim 2$ for the gas-poor models than for G8. The inner and outer halos (with respect to the bar $\mathrm{CR}$ ) follow the same trend, with the outer halo gaining more than the inner one.

The angular momentum of the disk is decreasing steadily, more sharply after $\sim 1$ Gyr, in all models. This decrease is a result of the $J$ loss by the inner disk over this 
time period. Subsequently, this $J$ increases very slightly in the gas-poor models and saturates in gas-rich models. The outer disk behavior traces (anti-)symmetrically that of the inner disk. The gas in the inner disk possesses small fraction of $J$ (i.e., in the stellar disk) even in gasrich models evolved here and rapidly loses it during the first Gyr, then stays flat for the remaining of the simulations. So, $J$ in the system flows from the inner disk to the outer disk and to the halo. The inner halo responds to this trend, largely because of the appearance of the 'ghost' bar in the DM there (Athanassoula 2005; Berentzen \& Shlosman 2006).

The role of gas in the overall balance of $J$ in the disk/halo system can be estimated from Fig. 9. Here we compare the direct input of $J$ into the stellar disk via the gravitational torques from the gas with the total flow of $J$ in the disk. The solid red lines show the input of $J$ by the gas to the stars by integrating over the torques in models with gradually reduced torques, G8BH, G8-50T and G8-100T. The solid pink lines describe the total $\Delta J$ in the stellar component. The red dotted line is identical to the red solid one but shifted downward for a direct comparison with the pink line. The $J$ input by the gas into the stars over 5 Gyr of the run is $\sim 13 \%$ of the stellar $J$ loss to the DM halo over this time. This relatively small input explains the near independence of the bar strength on the gas torques. However, as we discuss in the next section, the gas accumulation in the center resulting from the stellar bar has a profound effect on the evolution of the vertical structure in the bar.

\section{DISCUSSION: BAR EVOLUTION AND GAS}

The main goal of this work is to understand the effect of gas on stellar bar evolution. For this purpose we have run a series of models for stellar disks embedded in live DM halos, with various gas fractions ranging from $0 \%$ to $8 \%$ of the disk mass, with and without a growing central BH. In all of these models, the bar growth is barely affected by the gas presence - more gas-rich models display a marginally shorter rise time of the bar instability. The maximal values of the bar amplitude, $A_{2} \sim 0.5$, attained by the bar are very similar in all models. Past this maximum, the gas-poor models exhibit a plateau in $A_{2}$ for about $1 \mathrm{Gyr}$, followed by a sharp drop in the amplitude to $\sim 0.2$. This plateau gradually disappears with increasing $f_{\text {gas }}$ and its extent clearly anticorrelates with the CMC mass. Subsequently, the models differ in their evolution, ranging from a slight decline to a slight increase in $A_{2}$ - this evolution clearly separates gas-poor $\left(\mathrm{G} 0-\mathrm{G} 2 ; f_{\text {gas }}<3 \%\right)$ from gas-rich (G4-G8; $f_{\text {gas }}>3 \%$ ) models. In all models the stellar bar has survived during the computation time of $5 \mathrm{Gyr}$, although appears to be substantially weakened. Models with growing BHs show no significant difference in their evolution compared to models without the $\mathrm{BH}$.

Additional models testing the importance of various parameters for the dynamical and secular evolution of bars have been advanced. First, 25\% - 100\% of the gravitational torques, that the gas exerts on stars, have been removed in our most gas-rich model with $f_{\text {gas }}=8 \%$. This barely affected the $A_{2}$ curve. Second, we have added the gas 'force field' from our gas-rich model to the purely stellar model — the $A_{2}$ curve switched its behavior and closely followed the original gas model pre-

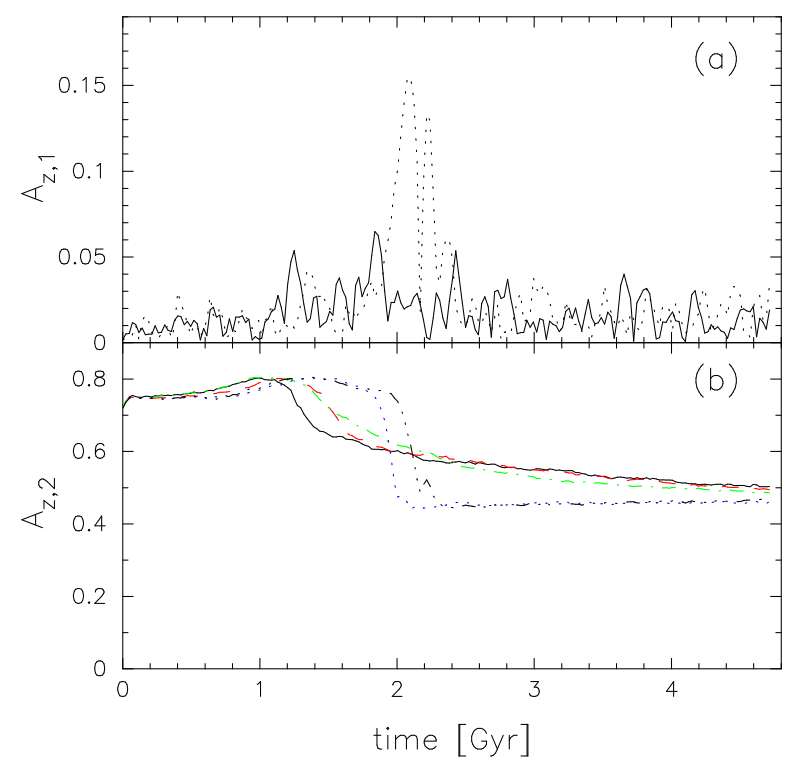

FIG. 10.- (a). The vertical asymmetry of the disk measured by $A_{\mathrm{z}, 1}$ Fourier coefficient. Shown are G0 (dotted black line) to G8BH (solid black) models. (b). The vertical 'strength' of the stellar bar measured by $A_{\mathrm{z}, 2}$ for $\mathrm{G} 8 \mathrm{BH}$ (solid black), $\mathrm{G} 6 \mathrm{BH}$ (dashed red), G4BH (dash-dotted green), G2BH (dotted blue) and G0 (black dash-dot-dot-dotted) models.

serving the same drop in $A_{2}$. Third, we run a number of pure stellar models, which have grown the $\mathrm{SBH}$ and the CMCs artificially, using their histories from the gas-rich model. No gravitational torques from the gas onto the stars have been present, but the bar strength has shown the same sharp decay. Fourth, pure stellar and gas-rich models have been run in hotter disks with initial $Q=1.8$ - those have shown qualitatively similar behavior, with bars reaching lower amplitudes but exhibiting the same drop in $A_{2}$. Finally, the amount of gas $J$ transferred to the stellar bar is small compared to the overall $J$ balance there.

The conclusion which emerges from these runs is that a direct input of the angular momentum from the gas into the stellar bar is not responsible for the sharp drop in its strength, even in the gas-rich models. It is most revealing that the pure stellar model shows no qualitatively different behavior from models with various gas fractions. Only with an additional analysis of the bar structure, the differences in the evolution between the gas-pure and gas-rich models start to emerge, as we discuss below.

\subsection{Pure stellar models: drop in the bar strength}

We now attempt to address the issue of what is the origin of the sharp weakening of the bar in G05-G8 and $\mathrm{G} 05 \mathrm{BH}-\mathrm{G} 8 \mathrm{BH}$ models with gas. The pure stellar model G0 acts here as the Rosette stone - the reason for the drop in its amplitude, $A_{2}$, is the increasing fraction of chaotic orbits within the bar (Martinez-Valpuesta \& Shlosman 2004). This behavior is triggered by the dynamical (buckling) instability, first detected in Combes \& Sanders (1981), and analyzed by Combes et al. (1990), Pfenniger \& Friedli (1991), Raha et al. (1991) and others, largely based on Toomre (1966) interpretation. The 


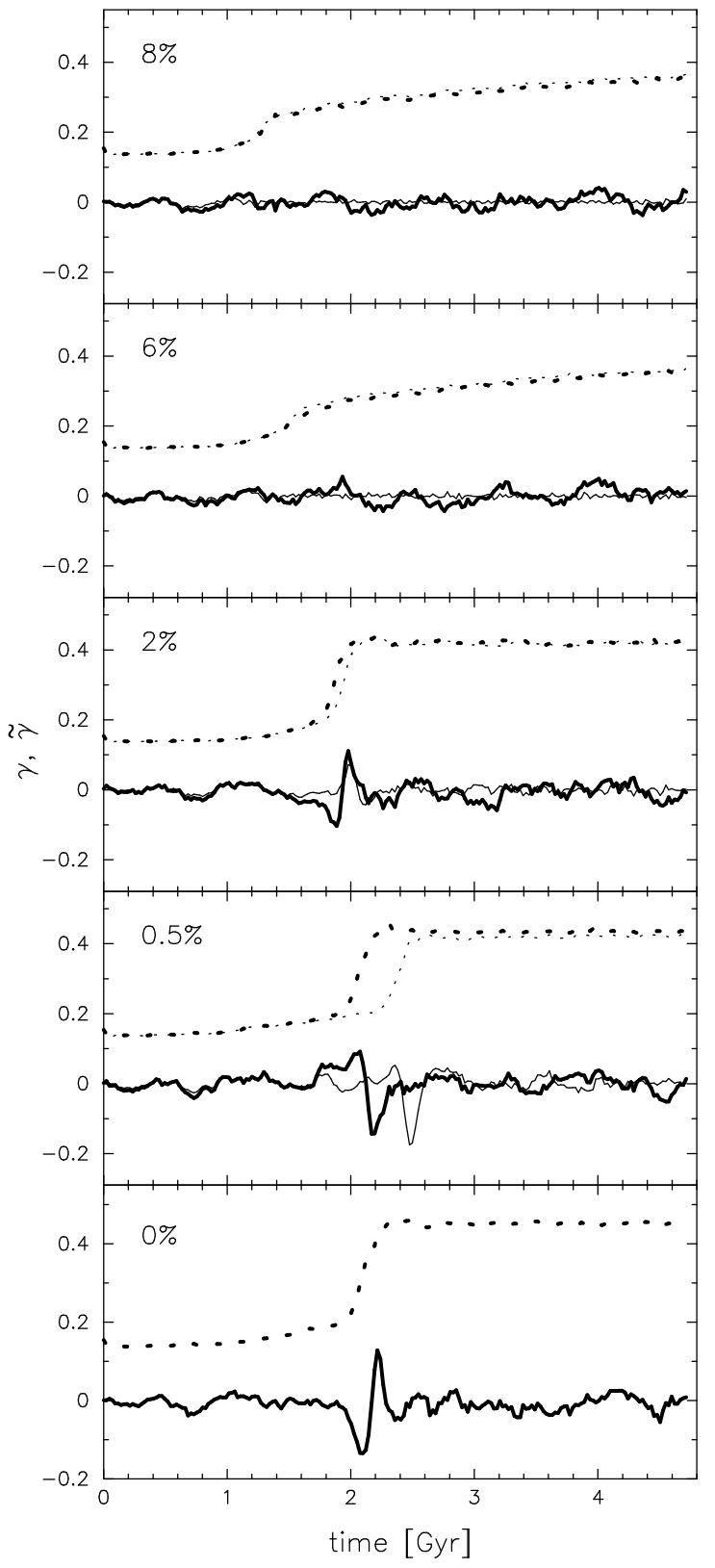

FIG. 11. - Vertical thickening of the bar $\gamma \equiv\left(1 / N_{0}\right) \Sigma_{\mathrm{i}} z_{\mathrm{i}} / r_{\mathrm{i}}$ (solid lines) and $\tilde{\gamma} \equiv\left(1 / N_{0}\right) \Sigma\left|z_{i} / r_{i}\right|$ (dashed lines) in models with $f_{\text {gas }}$ of $8 \%$ to $0 \%$ (from top to bottom). Thin and thick lines correspond to models with and without the SBH.

buckling instability is characterized by a spontaneous break of the symmetry with respect to the disk equatorial plane. It results in the vertical thickening of the bar which acquires a characteristic boxy/peanut shape, frequently observed in edge-on disks along the bar minor axis (e.g., Lütticke, Dettmar \& Pohlen 2000).

However, formation of the characteristic boxy/peanut shape of the inner bar does not necessitate the buckling instability. Friedli \& Pfenniger (1990) have shown that the near suppression of the vertical asymmetry in the bar still results in this characteristic shape, albeit established on a much longer secular timescale. They found that the bar thickening can be a direct consequence of the heating in the bar by its vertical inner Lindblad resonance
(ILR) and other lower resonances that scatter the stellar particles out of the disk plane.

These two alternatives for the bar to obtain the characteristic peanut shape can be reconciled. The initial growth of the stellar bar is accompanied by a strong increase in the chaotic motions in the $x y$-plane, especially in its outer part beyond the vertical ILR and close to CR. These motions are the prime reason for the outer bar dissolution and its overall weakening in the $x y$ plane - the bar actually shortens dramatically over a fraction of a Gyr. What is most important is that the role of the buckling is to accelerate the otherwise secular (vertical) heating to a dynamical timescale (Martinez-Valpuesta \& Shlosman 2004). Particles which normally confined to the bar equatorial plane are injected above it, allowing them to explore a larger configuration space. Hence one can distinguish between the buckling which is a dynamical instability and the action of the resonances which drive a slow vertical diffusion of the planar stellar orbits.

The model G0 exhibits just such a behavior that weakens the bar in the $x y$ plane but does not lead to a complete bar dissolution. It displays a strong vertical asymmetry at $\tau \sim 2-2.3$ Gyr as given by the (vertical) Fourier coefficient $A_{\mathrm{z}, 1}$ which has a maximum at this time (Fig. 10a) and the vertical bloating given by another Fourier coefficient in the vertical plane, $A_{\mathrm{z}, 2}$ (Fig. 10b). The initial growth of the stellar bar triggers two processes which appear to be nearly fatal for the bar itself. First, a larger fraction of stellar orbits in the bar becomes chaotic. The readily developing vertical ILR is located within the bar (typically half-way to the CR radius) and efficiently scatters (randomizes) the orbits in the bar vertical plane. Particles whose energy allows them to visit the outer part of the bar inevitably will cross the resonance region and will be affected most, thus dissolving the outer half of the bar. It is possible, but remains to be proven, that the first particles scattered above the disk plane act as 'seeds' for the (collective) buckling instability. This would explain why the appearance of the vertical ILR and the onset of the dynamic instability happen so close in time.

Strengthening of a stellar bar increases the importance of the vertical ILR and other lower resonances - this widens the associate resonance gaps in the characteristic diagrams. ${ }^{4}$ The ILR gap gives rise to specific symmetric/antisymmetric orbital families, called $\mathrm{BAN} / \mathrm{ABAN}^{5}$ correspondingly (Pfenniger \& Friedli 1991; Berentzen et al. 1998; Skokos et al. 2002; Martinez-Valpuesta et al. 2006). These orbits are the 3-D counterparts of the planar $x_{1}$ orbits introduced by Contopoulos \& Papayannopoulos (1980) and become populated when the planar orbits are destabilized. The BAN/ABAN orbits imprint their characteristic boxy/peanut shapes on the (inner) bar, in tandem with other 3-D families that originate at lower resonances. When the vertical symmetry in the bar is enforced, the population growth on these orbits is a slow secular process developing over many rotations of the bar.

\footnotetext{
4 These diagrams plot the $y$-intersection of a stellar orbit against the associated integral of motion, the Jacobi energy, $E_{\mathrm{J}}$ (e.g., Binney \& Tremaine 1987).

5 These are $2: 2: 1$ orbits, i.e., two radial oscillations for two vertical oscillations for one azimuthal turn.
} 


\subsection{Gas models: drop in the bar strength and quenching the bar buckling}

All models with the gas, shown in sections 3 and 4 , exhibit a drop in $A_{2}$ that is similar to the drop in the pure stellar model. Because the initial conditions for all these models are the same, and the evolution towards the peak in $A_{2}$ and the subsequent drop are nearly identical, it is tempting to assume that the same population of stellar orbits leaves the bar either as a result of the buckling or the action of the CMC. This population has been identified by Martinez-Valpuesta \& Shlosman (2004) as consisting mainly of chaotic orbits developing in the bar mid-plane. We do not pursue this line further here. The orbital nomenclatures are discussed in Berentzen et al. (1998) and in Patsis et al. (2002).

Evolution of the bar vertical shape is further complicated by the presence of the gas component. The amplitude of the vertical buckling, as measured by $A_{\mathrm{z}, 1}$, shows a two-fold behavior: gas-poor models exhibit a substantial buckling, while gas-rich models remain nearly symmetric (Fig. 10a). Berentzen et al. (1998) found that, in the presence of gas, the vertical instability in the bar is damped substantially. The new detail which emerges here is that, while the bar remains symmetric in the gasrich models, it nevertheless thickens. In the gas-poor models, the bars thicken abruptly, and subsequently remain unchanged, in the sense that the vertical swelling saturates immediately thereafter. In the gas-rich models, one can clearly distinguish two phases - an initial and fast swelling and a subsequent increase in the vertical thickness. This bimodal behavior is clearly displayed in Figs. 10b and 11. The former figure contrasts the vertical $A_{\mathrm{z}, 2}$ coefficient in $\mathrm{G} 0$ and gas models - the bulge forms nevertheless, but its boxy/peanut shape becomes progressively less prominent (as observed and quantified first by Berentzen et al. 1998, and confirmed by Athanassoula et al. 2005). Fig. 11 supports this conclusion through the measure of a new parameter $\gamma \equiv\left(1 / N_{0}\right) \sum_{i} z_{\mathrm{i}} / r_{\mathrm{i}}$ and its counterpart $\tilde{\gamma} \equiv\left(1 / N_{0}\right) \sum_{i}\left|z_{\mathrm{i}} / r_{\mathrm{i}}\right|$ for the stellar particles in the more gas-rich models, with and without the BHs. Here we sum over the cylindrical region of $0 \leq r \leq 10 \mathrm{kpc}$ and $|z| \leq 1 \mathrm{kpc}$, and $N_{0}$ is the number of stellar particles within this region used here for a normalization. These parameters quantify the (vertically) asymmetric and symmetric particle distributions respectively. They appear less noisy than $A_{\mathrm{z}, 1}$.

The trend in $A_{\mathrm{z}, 1}, A_{\mathrm{z}, 2}, \gamma$ and $\tilde{\gamma}$ that separates the gas-rich from the gas-poor models can be explained by the presence of the CMC. The growth of the CMC in our models is clearly linked to the gas, as the purely stellar model forms virtually no CMC ${ }^{6}$ On the other hand, the final mass of the CMC depends linearly on $f_{\text {gas }}$ in our models. Such massive CMCs will have immediate implications on the formation and shapes of galactic bulges and on the onset of the bar buckling. They destabilize the BAN/ABAN orbits, whose stable regions shrink towards the disk mid-plane - this damps the vertical asymmetry, reducing support for the boxy/peanut bulge shapes (Berentzen et al. 1998; Athanassoula et al. 2005). The increase in the stellar velocity dispersions proceeds both on dynamical and secular timescales, and is driven

\footnotetext{
6 The only contribution to the CMC in the stellar models comes from the stellar and DM ghost bars.
}

by resonant and non-resonant mechanisms, as we discuss below.

The action of the vertical resonances follows from the spatial part of the stellar distribution function, while its kinematic part drives the stellar vertical-to-radial velocity dispersion ratio, $\beta \equiv \sigma_{\mathrm{z}}^{2} / \sigma_{\mathrm{r}}^{2}$. The latter behavior is displayed in Fig. 12. We find that $\sigma_{\mathrm{r}}$ initially increases with time, which is related to the bar strengthening. At $\tau \sim 2-2.3 \mathrm{Gyr}$, for G0 and the gas-poor models, $\beta$ increases sharply from $\sim 0.3$ to $\sim 0.9$. This jump brings $\beta$ to nearly isotropic conditions, and its mere appearance signifies a sudden change in the vertical structure of the bar — stellar settling on the BAN/ABAN orbits. The subsequent gradual decrease in $\beta$ follows from a secular increase in $\sigma_{\mathrm{r}}$ and is related to the slow growth of the bar.

While an exact value of $\beta$, when the buckling instability is triggered, can depend on a particular model, within limits, we find that it lies around $\beta \sim 0.4$ for models presented here and in Martinez-Valpuesta et al. (2006). This value agrees well with that of Sellwood (1996) who found that some of his models remained unstable for up to $\beta \sim 0.4$.

The gas-rich models behave systematically different from the gas-poor models in Fig. 12, as much as they differ in Figs. 10 and 11. While $\beta$ still drops below the threshold of 0.4 , as the buckling develops, a steep growth of the CMCs in these models produces an equally abrupt heating of the stellar 'fluid' — an increase in the stellar dispersion velocities within the central kpc. This drives $\beta$ above the threshold and suppresses the buckling instability - a non-resonant effect. The comparative increase in $\sigma_{\mathrm{r}}$ prevents $\beta$ from growing to values attained in the gas-poor models. It is of a prime importance, that the vertical heating of $\sigma_{\mathrm{z}}$ is independent of $f_{\text {gas }}$, in tandem with the similar behavior of $A_{2}$ in all models. The subsequent secular increase in $\beta$ is driven by an increase in $\sigma_{\mathrm{z}}$ and is related to the action of the vertical resonances discussed earlier.

In a number of control runs (e.g., section 4), we have added the CMCs of various mass and compactness to the pure stellar model. The resulting $A_{\mathrm{z}, 1}$ and $A_{\mathrm{z}, 2}$ evolution remained two-fold and followed either the gas-poor or gas-rich models, although no gravitational torques from the gas onto the stars were involved. Models G0BH and G8BH250 behave as gas-poor, while G0BH80 and G0BH750 as gas-rich, displaying substantial or negligible vertical asymmetries correspondingly, but the overall thickening of the bar at the end of the simulations is similar to that in the pure stellar model.

\subsection{The bulge shape: effect of the gas}

The gradual loss of the characteristic shape of the bulge is correlated with increasing $f_{\text {gas }}$ in our models. The peanut shape is replaced by the boxy one and by an increasingly elliptical bulge (especially in the inner isodensities). In the gas-poor models, the increase in the velocity dispersion discussed earlier injects stellar particles above the disk. During the buckling instability, a spontaneous breakup of the vertical symmetry occurs, $\sigma_{z}$ increases and $\sigma_{\mathrm{r}}$ decreases (because the hottest particles in the $x y$ plane leave it). The vertical velocity dispersion (i.e., degree of freedom), $\sigma_{\mathrm{z}}$, in the disk is an adiabatic invariant and is affected by this dynamical instability. 


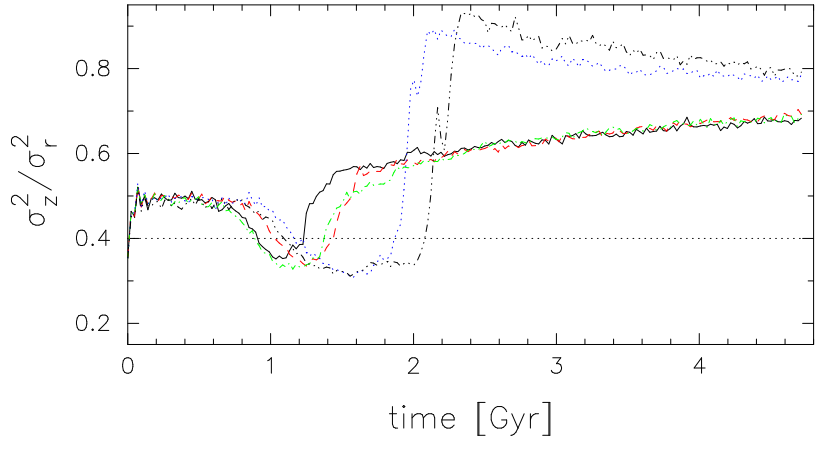

FIG. 12. - Evolution of $\beta \equiv \sigma_{\mathrm{z}}^{2} / \sigma_{\mathrm{r}}^{2}$ - the ratio of vertical dispersion velocities in G8BH (solid black), G6BH (dashed red), G4BH (dash-dotted green), G2BH (dotted blue) and G0 (black dash-dot-dot-dotted) models. $\beta$ is calculated using dispersion velocities within the central kpc. The dotted line at $\beta=0.4$ corresponds to the buckling instability limit for a wide range of models here and elsewhere.

For the gas-rich models, the steep increase in the CMC mass (see Figs. 2 and 3) pumps energy both in the vertical and horizontal planes - both $\sigma_{\mathrm{z}}$ and $\sigma_{\mathrm{r}}$ increase simultaneously. This heating of the stellar disk in the $x y$ and vertical planes is the result of a strong planar ILR which develops in the gas-rich models in addition to the vertical ILR that is present even in the gas-poor models. This was explicitly shown by Berentzen et al. (1998) for both types of models using identical initial conditions to those presented here. As a result, the Jacobi energy of stellar particles - normally a conserved quantity, increases abruptly, and particles are injected on 3-D orbits above the disk which are not trapped by the BAN/ABAN family. These orbits reduce support for the boxy/peanut-shape of the bulge which becomes progressively rounder with $f_{\text {gas }}$, as observed in our simulations.

An additional effect can contribute further to the damping of the vertical asymmetry in the gas-rich models. The condition for a linear vertical buckling of thin sheets with radial dispersion velocities has been analyzed by Toomre (1966). For pure collisionless systems, the instability saturates in the non-linear regime because of wave-particle and wave-wave damping (e.g., Sellwood, Nelson \& Tremaine 1998). In the presence of a substantial gas component, a strong damping originates in the two-fluid gas-stars, interaction. The source of this damping is in the overall response mismatch between the collisionless disk and the viscous gas. The difference in the stellar and gaseous responses can be noticed already in the linear regime, by applying the epicyclic approximation. Using Heller \& Shlosman (1996) notation, we follow the vertical oscillations at some radius $r$, irregardless of the excitation cause. While the stellar component follows the harmonic oscillator equation $\ddot{Z}_{1}(\tau)+\nu^{2} Z_{1}(\tau)=0$, the gas follows the damped oscillator equation, $\ddot{Z}_{1}(\tau)+\lambda \dot{Z}_{1}(\tau)+\nu^{2} Z_{1}(\tau)=0$, where the subscript ' 1 ' represents the first order terms, $\nu$ is the vertical epicyclic frequency, $\lambda(>0)$ is the damping coefficient in the gas, and the dots denote the time derivatives. The formal solution to the second equation is $Z_{1}(\tau)=A(\tau, \lambda) \cos (\omega \tau+\delta)$, where $A(\tau, \lambda)$ is the time-dependent (decaying) amplitude of the oscillation, $\omega=\omega(\nu, \lambda)$, and $\delta$ provides the phase-shift between the gaseous and stellar responses. This phase-shift depends explicitly on the $\lambda$ and disappears when $\lambda \rightarrow 0$.

The vertical oscillations which accompany the buckling instability are clearly visible in the numerical simulations of a pure stellar disk embedded in a live DM halo of Martinez-Valpuesta et al. (2006, see the accompanying animation) and we observe them here as well. The gas component buckles as well but immediately falls back to the disk mid-plane and remains unperturbed thereafter. The reason for this difference in behavior is the dissipation present in the gas - this prevents the BAN/ABAN orbits, that have sharp turns, from sustaining the gas.

Hence, the action of the CMC is to suppress the vertical buckling in stellar bars with larger gas fractions, above few percent of the disk mass, and to increase the stellar dispersion velocities. This weakens the boxy/peanut shape of the bulge and shortens the plateau in $A_{2}$. In the long run, the inner bar thickens additionally due to the secular action of the vertical resonances. So the development of the boxy/peanut bulges appears to be limited by the gas influx to the center in the gas-rich disks. This has been noted by Berentzen et al. (1998) using a lower resolution model, and by Athanassoula et al. (2005) using an analytical potential for the CMC. In the latter work, the CMC has been introduced after the boxy/peanut-shaped bulge has formed, while here we find that the growth of the CMC can weaken the orbital support for this shape in the first place. Of course the star formation will be important in altering the gas accumulation in the center and will affect the bulge morphology in some way. Future numerical modeling will quantify this process.

This means that the galactic stellar bars go through vigorous evolution not only in the equatorial disk plane but also in the plane normal to the disk. Geometrically thin bars in both planes readily develop a large population of chaotic orbits which 'leak' and thicken the bar over (at least) secular but sometime dynamical timescales. The gas inflow towards the central few hundred pc, while damping the vertical buckling, heats up the stellar disk - this is a vigorous heating which proceeds on dynamical and secular timescales. We find that $\beta$ still drops below 0.4 (Fig. 12), and that the vertical heating is not driven by the spiral structure in the disk as claimed by Debattista et al. (2006) - the heating exists even in a pure stellar model with a massive CMC which has no spiral structure. Furthermore, while the gas of course participates in the buckling itself, the gas layer remains thin and quickly collapses to the disk mid-plane - this result is not related to the isothermal EOS and persist for adiabatic gas as well. The only residual swelling of the gas layer comes from its response to the background stellar potential which becomes shallower with time.

The effect of the CMCs on the evolution of stellar bars in our simulations is important in determining the bar vertical and planar structure over the simulation time. Although we have limited the length of the evolution to $\sim 5$ Gyr, this is a substantial period of time to assess the immediate influence of the CMCs. Beyond shortening the plateau around the maximum in $A_{2}$, the main difference between gas-poor and gas-rich models lies in that former show bars which resume their growth after the period of buckling instability. The latter models display 
bars which exhibit a mild weakening.

The SBH masses in our simulations attain $\sim 0.3 \%-5 \%$ of the disk mass, depending on $f_{\text {gas }}$. From the observational point of view, this appears to be a factor of $\sim 10$ in excess of the $\mathrm{SBH}$ masses observed in disk galaxies. What is interesting is that even these large masses do not lead to a dissolution of stellar bars even over the time periods of 5 Gyr. This result is in sharp contrast with modeled bars of Friedli (1994) which decay completely over $1-2$ Gyr, but in agreement with Athanassoula et al. (2005) which report much more robust bars. One possible explanation lies in the absence of a DM halo in the former simulations. Under these conditions, the stellar bars develop faster, become stronger and have a larger fraction of chaotic orbits. The dissolving action of the $\mathrm{SBH}$ will be much more formidable in this case.

\subsection{Summary}

In summary, galactic bars in our simulations go through various stages of evolution, and we focus primarily on changes in the bar mid- and vertical planes. The pure stellar bars have been analyzed in this context in the literature (e.g., Chapter 1) — our goal was to understand how the gas presence modifies this evolution, in the range of $f_{\text {gas }} \lesssim 8 \%$. A large number of models of a two-component disk embedded in the live DM halo has been analyzed. We find a two-fold evolution and contrast the gas-poor, $f_{\text {gas }}<3 \%$ with the gas-rich, $f_{\text {gas }}>3 \%$ models. The exact dividing line, $f_{\text {gas }}$, between these groups can vary but the essence remains.

First, the angular momentum transfer from the gas to the stellar bar has no visible effect on the evolution of the bar strength in our models, beyond a well-known buildup of the CMC and the SBH there, contrary to Bournaud et al. (2005). We find that more massive CMCs shorten dramatically the extent of the plateau near the maxima of the bar strength. Second, all stellar bars thicken vertically, but the reason is two-fold - gas-poor models buckle while gas-rich models swell by preserving their symmetry. The vertical asymmetry (buckling) of the bar is damped in gas-rich models due to the forming CMC. Third, the vertical swelling starts earlier for the gas-rich disks and this effect increases with $f_{\text {gas }}$. Fourth, the vertical thickening of a stellar bar proceeds in two stages. Namely, the CMC heats up the central kpc in the stellar disk on a dynamical timescale, this stabilizes the bar against buckling, but puffs it up. This is followed by a slow (secular) stage of the bar thickening that complements the dynamical stage. Overall, the degree of the stellar bar thickening is practically independent of the gas fraction in the disk, for $f_{\text {gas }} \lesssim 8 \%$. Fifth, the action of the CMC leads to the formation of a weaker peanut and more elliptical bulge, in a contrast to the boxy/peanutshaped bulge forming in the gas-poor models, confirming the earlier result of Berentzen et al. (1998). The severity of this effect depends on the star formation process and the feedback from stellar evolution.

We acknowledge helpful conversations with Francoise Combes and Daniel Pfenniger. We thank Barbara Pichardo for helping with the initial stages of this project. This research has been partially supported by NASA/LTSA 5-13063, NASA/ATP NAG5-10823, HST/AR-10284 (to IS), and by NSF/AST 02-06251 (to $\mathrm{CH}$ and IS). IB acknowledges financial support by the project 'GRACE' I/80041 of the Volkswagen Foundation. IM-V is grateful for support by the Gruber Foundation.

\section{REFERENCES}

Athanassoula, E., Sellwood, J.A. 1983, Internal Kinematics and Dynamics of Galaxies, (Dordrecht: D. Reidel Publishing Co.), p. 203

Athanassoula, E. 1992, MNRAS, 259, 345

Athanassoula, E., Misiriotis, A. 2002, MNRAS, 330, 35

Athanassoula, E. 2003, MNRAS, 341, 1179

Athanassoula, E. 2005, in nonlinear Dynamics in Astronomy \& Physics, ed. S.T. Gottesman, J.R. Buchler \& M.E. Mahon, Ann. NY Acad. Sci., 1045, 168

Athanassoula, E., Lambert, J.C., Dehnen, W. 2005, MNRAS, 363, 496

Barnes, J., Hut, P. 1986, Nature, 324, 446

Begelman, M.C., Volonteri, M., Rees, M.J. 2006, MNRAS, 370, 289

Berentzen, I., Heller, C.H., Shlosman, I., Fricke, K. 1998, MNRAS, 300, 49

Berentzen, I., Athanassoula, E., Heller, C.H., Fricke, K.J. 2004, MNRAS, 347, 220

Binney, J., Tremaine, S. 1987, Galactic Dynamics, Princeton U. Press

Bournaud, F., Combes, F., Sememlin, B. 2005, MNRAS, 364, L18

Buta, R., Combes, F. 1996, Fund. Cosmic Phys., 17, 95

Combes, F., Sanders, R.H. 1981, A\&A, 96, 164

Combes, F., Debbasch, F., Friedli, D., Pfenniger, D. 1990, A\&A, 233,82

Contopoulos, G., Papayannopoulos, T. 1980, A\&A, 92, 33

Debattista, V.C., Mayer, L., Carollo, C.M., Moore, B., Wadsley, J., Quinn, T. 2006, ApJ. 645, 209

Dehnen, W. 2002, J. Comp. Phys., 179, 27

Englmaier, P., Shlosman, I. 2000, ApJ, 528, 677

Fall, S. M., Efstathiou, G. 1980, MNRAS, 193, 189

Ferrarese, L., Ford, H. 2005, Space Sci. Rev., 116, 523
Forbes, D.A., Norris, R.P., Williger, G.M., Smith, R.C. 1994, AJ, 107,984

Friedli, D., Pfenniger, D. 1990, in ESO/CTIO Workshop on Bulges of Galaxies, ed. B. Jarvis \& D.M. Terndrup (Garching: ESO), 265

Friedli, D., Martinet, L. 1993, A\&A, 277, 27

Friedli, D. 1994, in Mass-Transfer Induced Activity in Galaxies, ed. I. Shlosman (CUP), 268

Hasan, H., Norman, C.A. 1990, ApJ, 361, 69

Heller, C.H., Shlosman, I. 1994, ApJ, 424, 84

Heller, C.H. Shlosman, I. 1996, ApJ, 471, 143

Heller, C.H., Shlosman, I., Englmaier, P. 2001, ApJ, 553, 661.

Heller, C.H., Shlosman, I., Athanassoula, E. 2007, ApJ Lett., 657, L65

Ishizuki, S. Kawabe, R., Ishiguro, M., Okumura, K.S., Kasuga, T., Chikada, Y., Takashi, K. 1990, Nature, 334, 224

Jogee, S., Shlosman, I., Laine, S., Englmaier, P., Knapen, J.H., Scoville, N.Z., Wilson, C.D. 2002, ApJ, 575, 156

Jogee, S. 2006, Lect. Notes Phys. 693, 143

Kenney, J.D.P., Wison, C.D., Scoville, N.Z., Devereux, N.A., Young, J.S. 1992, ApJ, 395, L79

Knapen, J.H., Beckman, J.E., Heller, C.H., Shlosman, I., de Jong, R.S. 1995, ApJ, 454, 623

Knapen, J.H. 2005, A\&A, 429, 141

Kormendy, J., Kennicutt, R.C. 2004, ARA\&A, 42, 603

Lütticke, R., Dettmar, R.-J., Pohlen, M. 2000, A\&A, 145, 405

Lynden-Bell, D., Kalnajs, A.J. 1972, MNRAS, 157, L1

Maiolino, R., Alonso-Herrero, A., Anders, S., Quillen, A., Rieke, M.J., Rieke, G.H., Tacconi-Garman, L.E. 2000, ApJ, 531, 219

Martinez-Valpuesta, I., Shlosman, I. 2004, ApJ, 637, 214

Martinez-Valpuesta, I., Shlosman, I. Heller, C.H. 2006, ApJ, 613, L29

Norman, C.A., Sellwood, J.A., Hasan, H. 1996, ApJ, 462, 114 
Ostriker, J.P., Peebles, P.J.E. 1973, ApJ, 186, 467

Patsis, P.A., Skokos, Ch., Athanassoula, E. 2002, MNRAS, 337, 578

Pfenniger, D., Friedli, D. 1991, A\&A, 252, 75

Raha, N., Sellwood, J., James, R.A., Kahn, F.D. 1991, Nature, 352, 411

Sellwood, J.A. 1996, in Barred Galaxies, eds. R. Buta, D.A. Crocker \& B.G. Elmegreen (San-Francisco: ASP), 259

Sellwood, J.A., Nelson, R.W., Tremaine, S. 1998, ApJ, 506, 590

Sellwood, J.A. 2006, ApJ, 637, 567

Shen, J., Sellwood, J.A. 2004, ApJ, 604, 614

Shlosman, I., Frank, J., Begelman, M.C. 1989, Nature, 338, 45

Shlosman, I., Begelman, M.C., Frank, J. 1990, Nature, 345, 679
Shlosman, I., Noguchi, M. 1993, ApJ, 414, 474

Shlosman, I., Heller, C.H. 2002, ApJ, 565, 921

Shlosman, I. 2005, The Evolution of Starbursts, S. Huettemeister \& E. Manthey (eds.), Melville: AIP, 223

Skokos, Ch., Patsis, P.A., Athanassoula, E. 2002, MNRAS, 333, 847

Toomre, A. 1966, in Notes on the Summer School Program in Geophysical Fluid Dynamics at the Woods Hole Oceanographic Institution, Vol. 66, 111

Tremaine, S., Weinberg, M.D. 1984, MNRAS, 209, 729

Weinberg, M.D. 1985, MNRAS, 213, 451 